Check for updates

Cite this: RSC Adv., 2017, 7, 19479

Received 20th December 2016 Accepted 27th March 2017

DOI: 10.1039/c6ra28457d

rsc.li/rsc-advances

\section{Hybrid preparation of terbium(III)-doped mesoporous silica particles with calcium phosphates $\uparrow$}

\author{
T. Kataoka, ${ }^{a}$ K. Shiba, (D) *b L. Y. Wang, ${ }^{\text {a }}$ S. Yamada ${ }^{a}$ and M. Tagaya (D) ac
}

The preparation of terbium(III) $\left(\mathrm{Tb}^{3+}\right)$-doped mesoporous silica (Tb:MPS) particles and subsequent hybrid formation with calcium phosphate (CP) were proposed in this study. The surfactant/Tb:MPS particles with an initial doping concentration of $\mathrm{Tb}^{3+}$ ions to $(\mathrm{Si}+\mathrm{Tb}$ ) of $2 \mathrm{~mol} \%$ were synthesized by a sol-gel reaction based on the cooperative organization of tetraethoxysilane, terbium(III) chloride and cetyltrimethylammonium bromide. Subsequently, the Tb:MPS particles were hybridized with CP through precipitation to form CP/ Tb:MPS, where the formation was demonstrated by XRF and EDS analyses. The Tb:MPS showed a mesoporous structure which is typically seen in silica-surfactant mesostructured materials and the mesopores were preserved after precipitating the CP with crystalline phases. PL spectra were measured before and after the precipitation and some spectral changes were recognized. The PL spectral shapes due to the transitions of $\mathrm{Tb}^{3+}$ ions indicated that the $\mathrm{Tb}^{3+}$ ions were located inside the silica framework to interact with the siliceous $\mathrm{O}$ atoms. After the $\mathrm{CP}$ precipitation, the hybrid particles demonstrated higher quantum efficiency as well as longer PL life time, indicating the improved PL properties based on the $\mathrm{CP}-\mathrm{Tb}^{3+}$-silica hybrid states. The interfacial manipulation of the present $\mathrm{CP}-\mathrm{Tb}^{3+}$-silica hybrid allowed for unique material design in terms of its morphology and properties. This finding will be utilized for the encapsulation techniques for porous silica including with lanthanide ions and can be applicable for optical devices.

\section{Introduction}

Mesoporous materials have been prepared by the cooperative organization of surfactants and inorganic species; especially, the synthesis, characterization and applications of mesoporous silicas (MPSs) have been widely investigated. ${ }^{\mathbf{1 , 2}}$ The MPSs prepared by supramolecular templating methods are known to possess attractive features such as well-defined and controllable pore sizes, large surface areas, and reactive surfaces. ${ }^{3,4}$ The preparation of multifunctional MPSs is also a topic of interest toward a wider variety of applications. Thus, synthetic pathways for MPSs with various compositions have been explored for decades. ${ }^{5-10}$

Among various approaches, the incorporation of heteroatoms into the silica frameworks of MPSs has received much

${ }^{a}$ Department of Materials Science and Technology, Nagaoka University of Technology, 1603-1 Kamitomioka, Nagaoka, Niigata 940-2188, Japan

${ }^{b}$ World Premier International Research Center Initiative (WPI), International Center for Materials Nanoarchitectonics (MANA), National Institute for Materials Science (NIMS), 1-1 Namiki, Tsukuba, Ibaraki 305-0044, Japan. E-mail: SHIBA.Kota@nims. go.jp; Tel: $+81-29-860-4603$

${ }^{c}$ Top Runner Incubation Center for Academica-Industry Fusion, Nagaoka University of Technology, 1603-1 Kamitomioka, Nagaoka, Niigata 940-2188, Japan

$\dagger$ Electronic supplementary information (ESI) available: FT-IR spectra of the CTAB/Tb:MPS, CP/CTAB/Tb:MPS, CP/Tb:MPS and Tb:MPS. See DOI: $10.1039 / \mathrm{c} 6 \mathrm{ra} 28457 \mathrm{~d}$ attention. The framework structures can provide attractive features as a host organization by the charge-compensation of non-network oxygen species. ${ }^{\mathbf{1 1}}$ The lanthanide ion-doped MPSs demonstrated an improved thermal stability. ${ }^{12}$ The doping of cerium ions was accomplished for catalytic applications. ${ }^{\mathbf{1 3 , 1 4}}$ Europium (III) ion $\left(\mathrm{Eu}^{3+}\right)$ is known as an optical probe for the dopant site environment due to the luminescence properties. ${ }^{\mathbf{1 1 , 1 5}}$ We have reported the synthesis of $\mathrm{Eu}^{3+}$-containing MPSs which exhibited the efficient luminescence by the $\mathrm{Eu}^{3+}$ ions coordinated in the non-bridging oxygen atoms. ${ }^{16}$ Terbium(III) ion $\left(\mathrm{Tb}^{3+}\right)$ doped MPSs have also been synthesized and the presence of $\mathrm{Tb}^{3+}$ ions in the $\mathrm{O}-\mathrm{Si}-\mathrm{O}$ frameworks were investigated in detail. ${ }^{17}$ As a result, the obtained materials showed large specific surface areas. However, the efficient synthesis and luminescence of the $\mathrm{Tb}^{3+}$ doped MPSs by controlling the interactions between the doped ions and silica frameworks have not been reported. Thus, the effective control of $\mathrm{Tb}^{3+}$ ions at the near-surfaces in the silica frameworks is worth investigating.

Trivalent rare earth ions doped calcium phosphates (CPs) have been found to exhibit favorable photoluminescence properties. ${ }^{18-21}$ The CPs can display luminescence under visible excitation if it is doped with lanthanide ions, in which $\mathrm{Eu}^{3+}$ and $\mathrm{Tb}^{3+}$ are the most strongly emitting elements. ${ }^{22}$ Their luminescence is characterized by narrow emission bandwidths, high photochemical stability and long luminescent lifetime. ${ }^{23}$ Accordingly, Eu ${ }^{3+}$-doped ${ }^{24,25}$ and $\mathrm{Tb}^{3+}$-doped ${ }^{26,27}$ CPs have been 


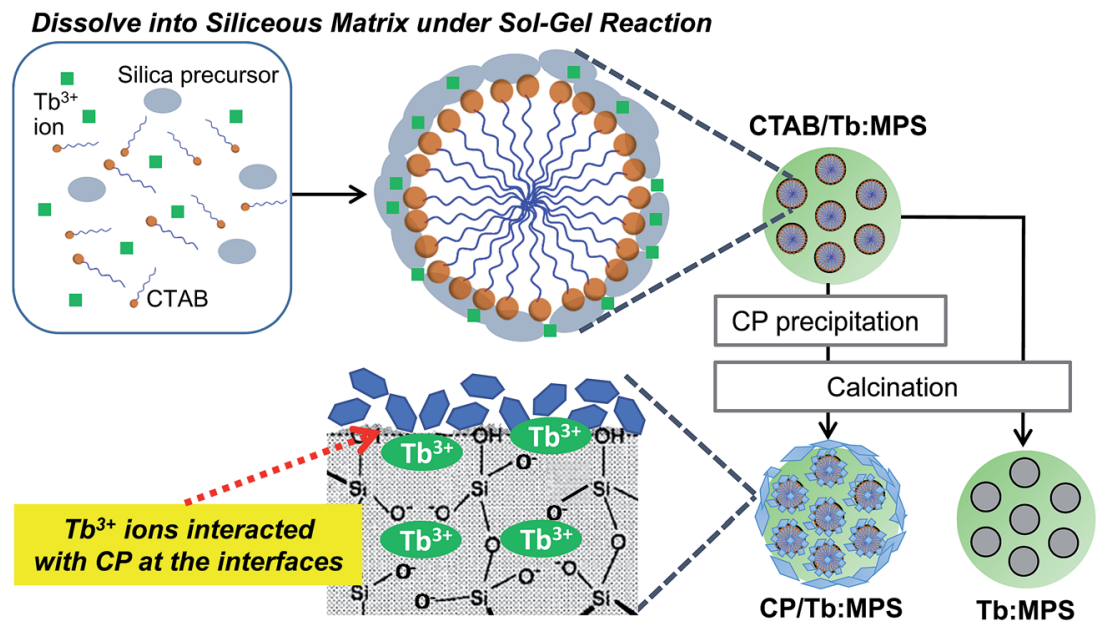

Scheme 1 Illustration for the preparation of the CTAB/Tb:MPS hybrid particles with CP. A possible Tb ${ }^{3+}$ ion state in the surface silica framework is also proposed.

studied. For the $\mathrm{Tb}^{3+}$-doped $\mathrm{CP}$, the near-surface calcium ions can be partially replaced by a small amount of $\mathrm{Tb}^{3+}$. Interestingly, the presence of an extremely low concentration of the doped $\mathrm{Tb}^{3+}$ on the surfaces greatly improved the luminescence, while the main physicochemical properties and bioactivity of the $\mathrm{CP}$ compounds were maintained. ${ }^{26}$ Therefore, the $\mathrm{Tb}^{3+}$ ions at the near-surfaces in MPS would easily interact with CP compounds to form the novel hybrids and the complexed $\mathrm{Tb}^{3+}$ ions at the interfaces should show the unique luminescent properties.

In this study, we introduce the synthesis and properties of the hybrid composed of the $\mathrm{Tb}^{3+}$ doped luminescent MPS particles and $\mathrm{CP}(\mathrm{CP} / \mathrm{Tb}: \mathrm{MPS})$ that induces the unique luminescence behaviors. The synthetic procedure of the $\mathrm{CP} /$ Tb:MPS hybrid particles is schematically shown in Scheme 1 in comparison with the $\mathrm{Tb}^{3+}$-doped MPS (Tb:MPS) particles. By a sol-gel reaction based on the cooperative organization of tetraethoxysilane, terbium(III) chloride and cationic surfactant (cetyltrimethylammonium bromide: CTAB), CTAB/ Tb:MPS particles were synthesized, and CP was precipitated on the particle surfaces. Then, the CTAB/Tb:MPS particles were calcined to resultantly obtain the mesoporous hybrid (CP/Tb:MPS). The unique interfacial interactions between $\mathrm{CP}-\mathrm{Tb}^{3+}$-silica at the hybrid interfaces were examined to explain the enhanced luminescence properties.

\section{Experimental}

\section{Preparation of Tb:MPS and CP/Tb:MPS}

$\mathrm{Tb}^{3+}$-doped MPSs were synthesized according to our previous report. ${ }^{17}$ All the reagents used here were special grades and were used as received without further purification. A $1.0 \mathrm{~g}(2.75 \mathrm{mmol})$ of cetyltrimethylammonium bromide (CTAB: Wako Co., Ltd.), $225 \mathrm{~g}$ of ultrapure water and $3.5 \mathrm{~mL}$ of $2.0 \mathrm{M}$ sodium hydroxide (NaOH: Wako Co., Ltd.) solution were mixed and then stirred at $80^{\circ} \mathrm{C}$ for $1 \mathrm{~h}$. A $5.515 \mathrm{~mL}(24.7 \mathrm{mmol})$ of tetraethoxysilane (TEOS: Tokyo Kasei Industries Co., Ltd.) and $15 \mathrm{~mL}$ of an $\mathrm{Tb}^{3+}$ aqueous solution containing $180 \mathrm{mg}$ of $\mathrm{TbCl}_{3} \cdot 6 \mathrm{H}_{2} \mathrm{O}$ (Wako Co., Ltd.) at the initial molar concentration of $\mathrm{Tb}$ to $(\mathrm{Si}+\mathrm{Tb})$ of $2.0 \mathrm{~mol} \%$ were admixtured, added and stirred at $80{ }^{\circ} \mathrm{C}$ for $4 \mathrm{~h}$. The resultant turbid solution was filtered. The separated solid was washed with ultrapure water and ethanol and then dried at $60^{\circ} \mathrm{C}$ for $12 \mathrm{~h}$. The sample is named as $\mathrm{CTAB} / \mathrm{Tb}: \mathrm{MPS}$, which was calcined in a furnace under air at $550^{\circ} \mathrm{C}$ for $6 \mathrm{~h}$ and the resultant sample is named as Tb:MPS, which was used as the reference for the following CP hybrids.

$\mathrm{K}_{2} \mathrm{HPO}_{4}(2.13 \mathrm{~g})$ was dissolved into deionized water (62.5 $\mathrm{mL}$ ), and the CTAB/Tb:MPS was added and dispersed into the phosphate solution. The $\mathrm{pH}$ value of the solution was adjusted to 9 using tetramethylammonium hydroxide (TMAOH: Wako Co., Ltd.). $62.5 \mathrm{~mL}$ of deionized water containing $\mathrm{CaCl}_{2} \cdot 2 \mathrm{H}_{2} \mathrm{O}$ $(3.67 \mathrm{~g})$ was added to the CTAB/Tb:MPS phosphate solution and vigorously stirred at the room temperature $3 \mathrm{~h}$. Then, the solution was centrifuged to sediment solid product and washed with ultrapure water and ethanol. The washed product was dried at $60{ }^{\circ} \mathrm{C}$ and the sample is named as $\mathrm{CTAB} / \mathrm{CP} / \mathrm{Tb}$ :MPS. Then, CTAB/CP/Tb:MPS was calcined in a furnace under air at $550{ }^{\circ} \mathrm{C}$ and the resultant sample is named as $\mathrm{CP} / \mathrm{Tb}$ :MPS.

\section{Characterization}

The elemental compositions were characterized by an X-ray fluorescence analysis (XRF: ZSX Primus II, Rigaku, Japan). The $\mathrm{XRF}$ analysis was performed using a sample pellet which was made by pressurizing sample powder without diluting it. The fundamental parameter method by the software (EZ scan program, Rigaku) was carried out for the semi-quantitative analysis. All the measurements and data analysis were conducted with an optional software (EZ scan program, Rigaku, Japan). Infrared spectra were recorded on a Fourier transform infrared spectrometer (FT-IR: JASCO Co., Ltd., FT/IR-4600ST). The FT-IR spectra were measured with a $\mathrm{KBr}$ pellet containing a small amount of sample powder. The weight ratio between sample powder and $\mathrm{KBr}$ was $1: 10$. All the spectra were recorded after subtracting a background spectrum of pristine $\mathrm{KBr}$. The 
measurement range, sampling time and resolution were 4000$500 \mathrm{~cm}^{-1}, 100$ and $2.0 \mathrm{~cm}^{-1}$, respectively. X-ray diffraction (XRD) patterns were recorded with a powder X-ray diffractometer (Smart Lab, Rigaku, Japan). XRD patterns were measured under the following conditions: X-ray source of CuK $\alpha$ line $(\lambda: 0.15418 \mathrm{~nm})$, voltage/current of $40 \mathrm{kV} / 30 \mathrm{~mA}$, scan speed of $5.0^{\circ} \mathrm{min}^{-1}$ and sampling width of $0.01^{\circ}$. The morphologies and elemental mapping were observed using a field emission scanning electron microscopy (FE-SEM: SU8230, Hitachi High-Technologies Co., Ltd.) equipped with an energy dispersive spectroscopy (EDS: XMax $^{N}$ large area silicon drift detector, Oxford Instruments). Nitrogen adsorption and desorption isotherms were measured at $77 \mathrm{~K}$ on a BELSORP-mini II instrument (Microtrac/BEL Co., Ltd.). Prior to the measurement, the samples were degassed under vacuum at $393 \mathrm{~K}$ for $4 \mathrm{~h}$. The surface area was evaluated by the Brunauer-Emmett-Teller (BET) method. ${ }^{28}$

Photoluminescence properties were evaluated by a photoluminescence spectroscopy and microscopy. The excitation and luminescence spectra were recorded on a FP-8500 spectrophotometer (JASCO Co., Ltd.) with the monitored luminescence at $544 \mathrm{~nm}$ and excitation wavelength at $369 \mathrm{~nm}$ from Xe lamp under room temperature (atmosphere: air, excitation-slit/detection-slit: $2 \mathrm{~nm} / 2 \mathrm{~nm}$, measure time: $0.1 \mathrm{~s}$, step width: $1.0 \mathrm{~nm}$, sample weight: $150 \mathrm{mg}$, shape: pellet), and the detection was used by photomultiplier tube. The fluorescent microscope images were obtained using a luminescence microscope (OLYMPUS Co., Ltd., CKX41N-FL, excitation wavelength: $360-400 \mathrm{~nm}$, exposure time: $100 \mathrm{~ms}$, sensitivity: ISO 400) through the emission source (OLYMPUS Co., Ltd., U-RFLT50).

The internal quantum efficiency $\left(\eta_{\text {int }}\right)$ was measured using an integrating sphere (internal diameter: $60 \mathrm{~mm}$ ). The excitation and luminescence spectra were also recorded by the above spectrophotometer with each monitored luminescence maxima and excitation wavelength. The incident, scattering, and luminescence light intensity spectra were measured, and their integrated peak intensities were calculated and abbreviated $I_{0}, I_{1}$ and $I_{2}$, respectively. Thus, $\eta_{\text {int }}$ was calculated by the eqn (1).

$$
\eta_{\text {int }}=I_{2} /\left(I_{0}-I_{1}\right) \times 100
$$

The decay kinetics was also recorded by the above spectrophotometer with the same excitation and luminescence wavelength, which can be explained with an exponential function. The excitation was measured on a wavelength at $369 \mathrm{~nm}$ from a Xe flash lamp $(150 \mathrm{~W})$, and the decay of the luminescence intensity was detected on a wavelength at $544 \mathrm{~nm}$. The data plot time was $0.05 \mathrm{~ms}$ using a successive rotation chopper. The measured decay was recorded by a numerical fitting to an exponential function based on the eqn (2).

$$
I(t)=I(0) \exp (-t / \tau)
$$

where the $I(t)$ and $I(0)$ are the luminescence intensity at time $(t)$ and starting point $(t=0)$, and the $\tau$ is luminescence life time in the decay process.

\section{Results and discussion}

As shown in Table 1, the elemental concentrations, i.e. $\mathrm{Ca} / \mathrm{P}$ and $\mathrm{Tb} /(\mathrm{Si}+\mathrm{Tb})$, were determined and calculated by the XRF results. Although most of the carbons were removed during the precipitation and washing process, some carbon was still remained even after the calcination. It would be due to the residual CTAB. The resulting $\mathrm{Tb} /(\mathrm{Tb}+\mathrm{Si})$ concentrations were almost the same between the samples. The resulting $\mathrm{Tb} /(\mathrm{Tb}+$ $\mathrm{Si}$ ) concentration was 1.8-2.0 times larger than those in the initial solution composition, suggesting the decrease in the $\mathrm{Si}$ content of the particles by the Tb added synthetic process. The $\mathrm{Ca}$ and $\mathrm{P}$ elements were seen only in the case of the CP hybrid and the $\mathrm{Ca} / \mathrm{P}$ ratio was not changed after the calcination.

In the FT-IR spectra (see Fig. S1 in ESI $\dagger$ ), the absorption bands originated from the $\mathrm{Si}-\mathrm{O}-\mathrm{Si}$ asymmetric and symmetric stretching were observed at around 1070 and $1225 \mathrm{~cm}^{-1}$ and these spectral shapes were almost same irrespective of the doping. The characteristic bands seen at around 1480, 2854 and $2925 \mathrm{~cm}^{-1}$ can be assigned to the $\mathrm{C}-\mathrm{H}$ stretching and bending of the CTAB that apparently decreased after the calcination. The present hybrid states would suppress the complete removal of the carbon compounds, while the carbon compounds were completely removed in the case of Tb:MPS. Based on the previous reports on the FT-IR spectroscopic studies, ${ }^{29,30}$ in the $\mathrm{CP} / \mathrm{CTAB} / \mathrm{Tb}$ :MPS and CP/Tb:MPS, the several absorption bands appeared at around 1090-1030, 600 and $560 \mathrm{~cm}^{-1}$ are attributed to $\mathrm{P}-\mathrm{O}$ stretching of phosphate groups, suggesting that the $\mathrm{CP}$ formation on Tb:MPS was verified.

Fig. 1 shows the XRD patterns of the particles at lower and higher $2 \theta$ regions. In the CTAB/Tb:MPS and Tb:MPS particles, the diffractions indexed as 100, 110 and 200 reflections of a hexagonal structure were observed (Fig. 1(a)). With the calcination of CTAB/Tb:MPS, the shapes of each diffraction pattern were preserved while slight shift to higher angles was recognized, indicating the pore contraction by the condensation among residual silanol groups of the silica framework. The Tb$\mathrm{O}$ bond length was expected to be longer than that of $\mathrm{Si}-\mathrm{O}$, resulting in the enlargement of the unit cell in the presence of $\mathrm{Tb}^{3+}$ as compared to the bare mesoporous silicas. This was also demonstrated by the metal incorporation into the MCM-41 framework. ${ }^{32}$ With the CP formation, the $d_{100}$ - spacing value increased and the diffraction peak shape was broadened. The $\mathrm{CP}$ nucleation and crystal growth at the mesopore surfaces would induce the pore dilatation.

In the higher-angle regions (Fig. 1(b)), the CTAB/Tb:MPS and Tb:MPS do not display any detectable diffraction that can be assigned to terbium oxide, for example. Thus, $\mathrm{Tb}^{3+}$ ions should be incorporated into the amorphous silica networks. It was found that the $\mathrm{Tb}^{3+}$-doping into the silica frameworks can expand the mesopores and then rearrange the $\mathrm{Si}-\mathrm{O}-\mathrm{Si}$ networks without any crystalline impurities (e.g., terbium oxides and silicates), which would be based on the interactions with $\mathrm{Tb}^{3+}$ ions. With the $\mathrm{CP}$ formation, some $\mathrm{CP}$ crystalline phases (hydroxyapatite $\left(\mathrm{Ca}_{10}\left(\mathrm{PO}_{4}\right)_{6}(\mathrm{OH})_{2}\right.$, ICDD 00-009-0432), monetite $\left(\mathrm{CaHPO}_{4}, \quad\right.$ ICDD 01-071-1759), calcium pyrophosphate 
Table 1 Chemical compositions of the CTAB/Tb:MPS, CP/CTAB/Tb:MPS, CP/Tb:MPS and Tb:MPS particles, their Ca/P ratios and Tb/(Tb + Si) concentrations

\begin{tabular}{|c|c|c|c|c|c|c|c|c|c|}
\hline & $\mathrm{C}(\mathrm{mol} \%)$ & $\mathrm{O}(\mathrm{mol} \%)$ & Si (mol\%) & $\mathrm{P}(\mathrm{mol} \%)$ & $\mathrm{K}(\mathrm{mol} \%)$ & $\mathrm{Ca}(\mathrm{mol} \%)$ & $\mathrm{Tb}(\mathrm{mol} \%)$ & $\mathrm{Ca} / \mathrm{P}$ & $\begin{array}{l}\mathrm{Tb} / \\
(\mathrm{Tb}+\mathrm{Si})(\mathrm{mol} \%)\end{array}$ \\
\hline CTAB/Tb:MPS & 32.9 & 46.2 & 19.5 & 0.0 & 0.0 & 0.0 & 1.4 & - & 4.3 \\
\hline CP/CTAB/Tb:MPS & 2.6 & 63.6 & 1.5 & 13.3 & 1.1 & 17.0 & 0.9 & 1.28 & 3.7 \\
\hline CP/Tb:MPS & 2.5 & 63.8 & 2.0 & 13.1 & 1.2 & 16.5 & 0.9 & 1.26 & 4.8 \\
\hline Tb:MPS & 14.4 & 59.3 & 24.6 & 0.0 & 0.0 & 0.0 & 1.1 & - & 4.1 \\
\hline
\end{tabular}

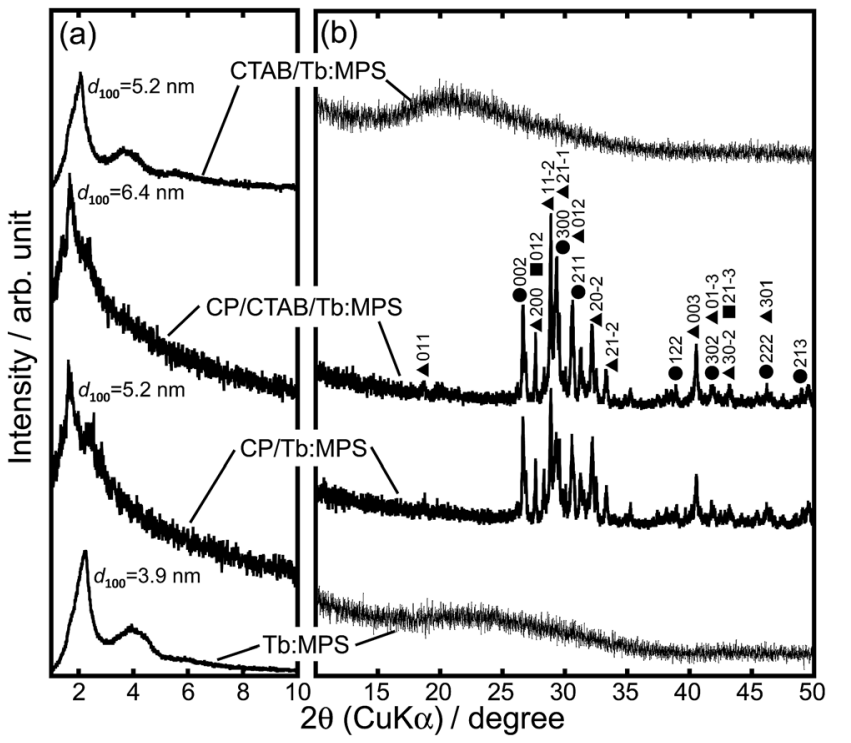

Fig. 1 XRD patterns of the CTAB/Tb:MPS, CP/CTAB/Tb:MPS, CP/ Tb:MPS and Tb:MPS particles at (a) lower and (b) higher $2 \theta$ regions $(\bullet$ : hydroxyapatite (ICDD 00-009-0432), $\boldsymbol{\Delta}$ : monetite (ICDD 01-0711759); : calcium pyrophosphate (ICDD 01-075-2756)).
$\left(\mathrm{Ca}_{2} \mathrm{P}_{2} \mathrm{O}_{7} \cdot 4 \mathrm{H}_{2} \mathrm{O}\right.$, ICDD 01-075-2756)) were observed, allowing the presence of significant amounts of crystalline CP phases. Considering only single phase formation of hydroxyapatite without CTAB/Tb:MPS particles, the cationic surfactant CTAB, $\mathrm{Tb}^{3+}$ ions and mesopore surfaces would affect the crystalline CP phases. Here, it should also be mentioned that both $\mathrm{Ca}^{2+}$ and $\mathrm{Tb}^{3+}$ ions can provide a similar coordination environment and hardness, ionic radii, and geometric characteristics. Thus, the $\mathrm{Tb}^{3+}$ ion in the silica phase would partially be incorporated into the CP phases at the precipitation process. Therefore, the interfacial reactions between the CTAB/Tb:MPS and CP occurred in the precipitation and calcination processes. To simply demonstrate the properties of $\mathrm{CP} / \mathrm{Tb}$ :MPS by comparing to those of Tb:MPS, the samples after the calcination will be discussed hereafter.

Fig. 2(a) shows the $\mathrm{N}_{2}$ adsorption and desorption isotherms of the CP/Tb:MPS and Tb:MPS particles. The shape of the isotherms for $\mathrm{CP} / \mathrm{Tb}$ :MPS is categorized into type III according to the IUPAC classification, whereas that for Tb:MPS is categorized into typical type IV, suggesting the mesopore formation. As shown in Table 2, the BET surface area $\left(S_{\mathrm{BET}}\right)$ decreased with the CP formation. The Barrett-Joyner-Halenda (BJH) pore size distributions ${ }^{33}$ indicated the contraction of the pore diameter (a)

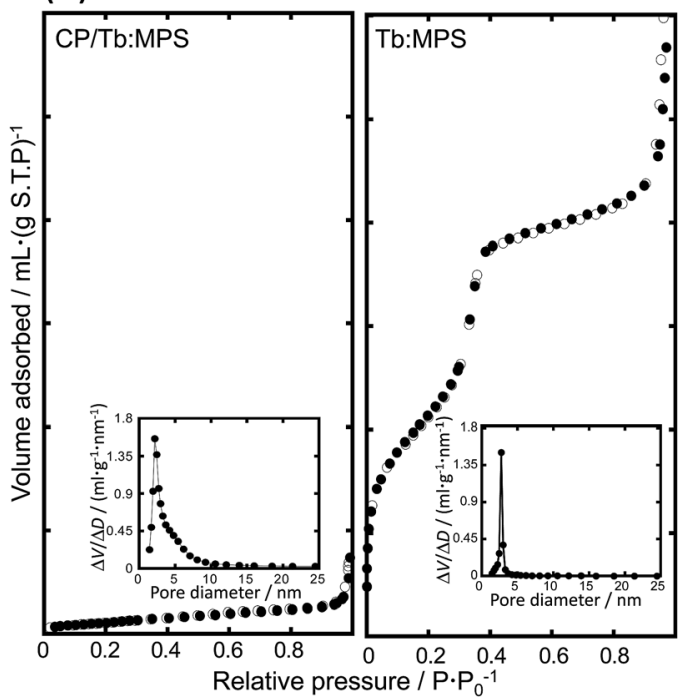

(b)

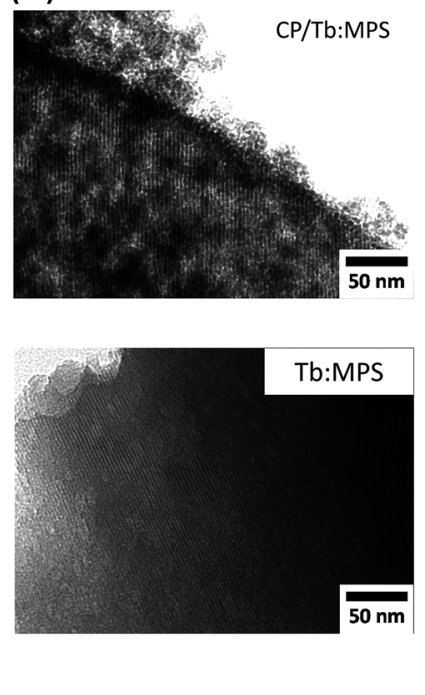

Fig. 2 (a) Nitrogen adsorption ) and desorption $(O)$ isotherms and (inset) corresponding pore size distributions of the CP/Tb:MPS and Tb:MPS particles, and (b) their TEM images of the mesopores. 
Table $2 S_{\mathrm{BET}}, r_{\mathrm{BJH}}, \eta_{\text {int }}$ and $\tau$ of the CP/Tb:MPS and Tb:MPS particles

\begin{tabular}{lrlll}
\hline & $S_{\mathrm{BET}}\left(\mathrm{m}^{2} \mathrm{~g}^{-1}\right)$ & $r_{\mathrm{BJH}}(\mathrm{nm})$ & $h_{\text {int }}(\%)$ & $t(\mathrm{~ms})$ \\
\hline CP/Tb:MPS & 48 & 2.2 & 4.4 & 5.0 \\
Tb:MPS & 894 & 2.7 & 2.8 & 1.6
\end{tabular}
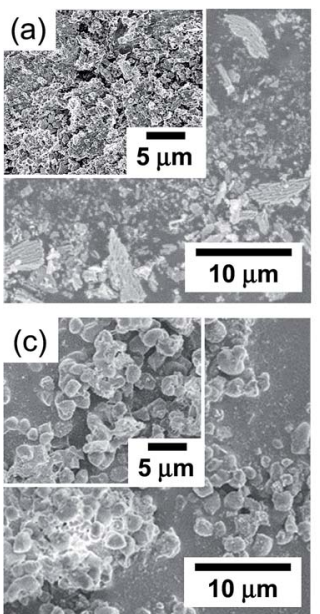

(b)

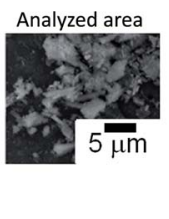

(d)

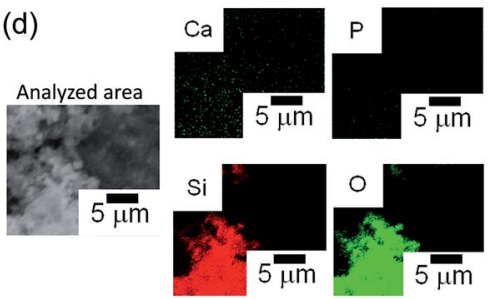

Fig. 3 ( $a$ and $c)$ FE-SEM and ( $b$ and d) FE-SEM/EDS mapping (element: $\mathrm{Ca}, \mathrm{P}, \mathrm{Si}$ and $\mathrm{O}$ ) images of ( $\mathrm{a}$ and $\mathrm{b}$ ) CP/Tb:MPS and ( $\mathrm{c}$ and $d$ ) Tb:MPS particles.

with the formation, although the distribution width was broadening. Thus, the mesopore entrances on the particle surfaces were encapsulated by the nucleated CP and CP crystals, which result in the decrease in the $S_{\mathrm{BET}}$. The broadening of the X-ray diffraction pattern and pore distribution suggested the presence of less ordered structure, but the mesopore structures were still retained, being also supported by the previous reports. ${ }^{17,31}$

In order to investigate the hybrid nanostructures, we observed the TEM images shown in Fig. 2(b). All the TEM images clearly exhibited the mesopore arrangements and the pore diameters were approximately estimated to be $1.5-4.0 \mathrm{~nm}$ from the images. In CP/Tb:MPS, the precipitated CP crystallites were significantly covered on the Tb:MPS surfaces without closing the mesopores, while the silica framework exhibited the disordering of the mesopore structures (i.e., worm-like pores). Thus, these results are in agreement with those obtained by the $\mathrm{XRD}$ and $\mathrm{N}_{2}$ adsorption/desorption measurements.

In the FE-SEM images (Fig. 3(a) and (c)), the CP/Tb:MPS exhibited irregular shapes with the smaller particles, whereas the Tb:MPS had the spherical shapes, indicating that the CP precipitation on the silica frameworks induced the morphological changes with collapsing the shapes. In the EDS mapping images (Fig. 3(b) and (d)), the CP/Tb:MPS particle shapes corresponded with the $\mathrm{Ca}, \mathrm{P}, \mathrm{Si}$ and $\mathrm{O}$ mapping areas and the $\mathrm{Si}$ intensity was relatively week, indicating the $\mathrm{CP}$ precipitation on the silica surfaces. In contrast, no detectable $\mathrm{Ca}$ and $\mathrm{P}$ elements was observed in the case of the Tb:MPS particles. Therefore, the Tb:MPS particles were successfully hybridized with the CP phases.

Fig. 4(a) and (b) show the excitation and photoluminescence spectra. These spectral shapes are almost same irrespective of the CP formation. Thus, no reflection of the electronic transitions of $\mathrm{Tb}^{3+}$ indicates the same local $\mathrm{O}$ atom environments in the silica host matrix. The excitation spectra exhibited the peaks associated with $4 \mathrm{f}-4 \mathrm{f}$ transitions of $\mathrm{Tb}^{3+}$ ion (Fig. 4(a)). The features are mainly assigned to the excitations based on the ${ }^{5} \mathrm{~L}_{J}$ $(J=10,9,7)$ levels from the ground state of $\mathrm{Tb}^{3+}$ ion..$^{34,35}$ The luminescence intensity under the excitation at $369 \mathrm{~nm}$ by the $4 \mathrm{f}-4 \mathrm{f}$ transition from ${ }^{5} \mathrm{~L}_{10}$ level to the ground state is relatively stronger in the observed peaks.

The luminescence spectra showed the narrow peaks assigned to the $\mathrm{f}-\mathrm{f}$ transitions of the $\mathrm{Tb}^{3+}$ ions (Fig. 4(b)). The luminescence transitions are assigned to those from ${ }^{5} \mathrm{D}_{4} \rightarrow{ }^{7} \mathrm{~F}_{J}$ $(J=6,5,4,3)^{36}$ as usual for $\mathrm{Tb}^{3+}$ luminescence, being centered at 490, 544, 587 and $623 \mathrm{~nm}$, respectively. The green luminescence is seen in the microscope image (Fig. 4 (inset)), and the

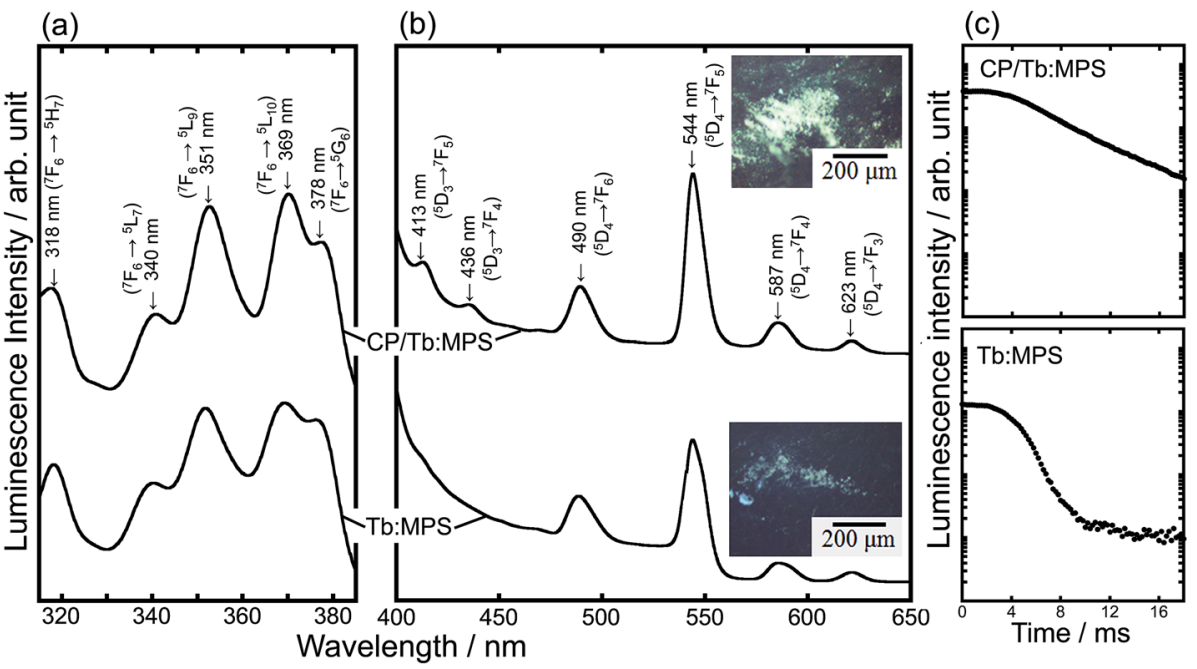

Fig. 4 (a) Excitation and (b) photoluminescence spectra of the CP/Tb:MPS and Tb:MPS particles and their (inset) fluorescent images, and (c) fluorescence intensity decay plots. 
color is also observed in the other $\mathrm{Tb}^{3+}$-doped systems (e.g., $\mathrm{Tb}^{3+}$-doped $\left.\mathrm{SiO}_{2}-\mathrm{PVA}^{37}\right)$. By the $\mathrm{CP}$ hybrid, the luminescence peaks due to ${ }^{5} \mathrm{D}_{3} \rightarrow{ }^{7} \mathrm{~F}_{J}(J=4,5)$ were enhanced (Fig. 4(b)) and the apparent luminescence intensities increased (Fig. 4 (inset)). In fact, as shown in Table 2, the $\eta_{\text {int }}$ significantly increased with the $\mathrm{CP}$ formation, although the residual carbon compounds of CTAB would affect the luminescence properties.

To understand the luminescence relaxation, the decay kinetics of the excited states was investigated as shown in Fig. 4(c) and Table 2. The decay kinetics was fitted with an exponential function. The CP precipitation extended $\tau$, even though only the silica framework exhibited the longer $\tau$ of the $\mathrm{Tb}^{3+}$ as compared with the previous report. ${ }^{34}$ The $\tau$ clearly reveals the dispersion of the $\mathrm{Tb}^{3+}$ ions into the silica frameworks and the encapsulation of $\mathrm{CP}$ induce the preferred interfacial interactions between the $\mathrm{CP}-\mathrm{Tb}^{3+}$ silica to exhibit the longer $\tau$, suggesting the interfacial design for the unique luminescence properties. At the interfaces between CP and Tb:MPS, the preferred $\mathrm{CP}-\mathrm{Tb}^{3+}$-silica interactions would be present. Then, the near-surface $\mathrm{Tb}^{3+}$ ions in the silica phases were partially incorporated into the $\mathrm{CP}$ phases. Such an incorporation would change the luminescence properties.

$\mathrm{The} \mathrm{Eu}^{3+}$ ion states in the silica frameworks have been investigated in our previous report. ${ }^{11} \mathrm{The} \mathrm{Eu}^{3+}$ ions were located inside the structure to electrostatically interact with the siliceous $\mathrm{O}$ atoms. Under the sol-gel process, the $\mathrm{Eu}^{3+}$ ions would be homogeneously dissolved to resultantly prevent aggregation among the $\mathrm{Eu}^{3+}$ ions in the MPS formation. A homogeneous sol containing CTAB, silica precursor and $\mathrm{Tb}^{3+}$ was used in the synthetic process. Although the mesopores with the large surface area effectively adsorb water molecules, the $\mathrm{Tb}^{3+}$ doped inside the framework would little interact with the water molecules. Therefore, it is suggested that the $\mathrm{Tb}^{3+}$ ions homogeneously diffused inside the non-crystalline silica frameworks, and would be stabilized by the chargecompensation of the non-network oxygen species $\left(e . g\right.$., $\left.\mathrm{Si}^{-} \mathrm{O}^{-}\right)$. The near-surface $\mathrm{Tb}^{3+}$ ions easily interact with the hydroxyl groups of the adsorbed water molecules or silanol groups. The presence of high energy stretching vibrations of the hydroxyl groups causes the luminescence quenching by a non-radiative decay mechanism. In this study, the interfacial design through the encapsulation by CP induced the unique luminescence properties. This finding provides a merit in the use of the interfacial design between the $\mathrm{CP}-\mathrm{Tb}^{3+}$-silica hybrid states for the activity of lanthanide ions. Here, the ionic radius of $\mathrm{Tb}^{3+}(1.04 \AA)$ is larger than that of $\mathrm{Si}^{4+}(0.41$ $\AA)$ and same as $\mathrm{Ca}^{2+}(1.14 \AA)$. In the present case, the near-surface $\mathrm{Tb}^{3+}$ ions in the silica phases were partially incorporated into the $\mathrm{CP}$ phases. Such an incorporation could be explained by the sector zoning model in minerals. ${ }^{38,39}$ Therefore, the silica frameworks and subsequent encapsulation of $\mathrm{CP}$ induced the preferred interfacial interactions between the $\mathrm{CP}-\mathrm{Tb}^{3+}$-silica for the unique luminesce properties. After achieving the efficient luminescent properties by the further investigation, the hybrids will be utilized as bio-imaging materials based on the biocompatibility of CP.

\section{Conclusions}

The preparation of $\mathrm{Tb}^{3+}$-doped MPS particles and subsequent hybrid formation with $\mathrm{CP}$ were investigated. The $\mathrm{CP}$ was precipitated on the CTAB/Tb:MPS particles which were synthesized by a sol-gel reaction of tetraethoxysilane in the presence of terbium(III) chloride and CTAB. The CTAB containing hybrid was converted to mesoporous solid by the calcination and the mesopores were preserved even after precipitating the $\mathrm{CP}$ with crystalline phases. The formation of the present CP/Tb:MPS hybrid particles were confirmed by XRF and EDS analyses. PL spectra were also measured before and after the CP precipitation and some spectral changes were recognized. The PL spectral shapes due to the transitions of $\mathrm{Tb}^{3+}$ ions indicated that the $\mathrm{Tb}^{3+}$ ions were located inside the silica frameworks, interacting with the siliceous $\mathrm{O}$ atoms. After the $\mathrm{CP}$ precipitation, the hybrid particles demonstrated higher quantum efficiency as well as longer PL life time, suggesting the improved PL properties based on the $\mathrm{CP}-\mathrm{Tb}^{3+}$-silica hybrid states. This finding will be utilized for the encapsulation techniques of the porous silica including lanthanide ions and can be applicable to optical devices.

\section{Acknowledgements}

This study was supported by a grant from the Japan Society for the Promotion of Science (JSPS) KAKENHI (Grant-in-Aid for Young Scientists (A), Grant No. 26709052).

\section{Notes and references}

1 T. Yanagisawa, T. Shimizu, K. Kuroda and C. Kato, Bull. Chem. Soc. Jpn., 1990, 63, 988-992.

2 C. T. Kresge, M. E. Leonowicz, W. J. Roth, J. C. Vartuli and J. S. Beck, Nature, 1992, 359, 710-712.

3 R. I. Nooney, D. Thirunavukkarasu, Y. Chen, R. Josephs and A. E. Ostafin, Chem. Mater., 2002, 14, 4721-4728.

4 M. Ogawa, K. Kato and N. Shimura, Bull. Chem. Soc. Jpn., 2009, 82, 121-125.

5 I. I. Slowing, B. G. Trewyn and V. S. Y. J. Lin, J. Am. Chem. Soc., 2007, 129, 8845-8849.

6 J. S. Beck, J. C. Vartuli, W. J. Roth, M. E. Leonowicz, C. T. Kresge, K. D. Schmitt, C. T.-W. Chu, D. H. Olson, E. W. Sheppard, S. B. McCullen, J. B. Higgins and J. L. J. Schlenker, J. Am. Chem. Soc., 1992, 114, 10834-10843.

7 M. Tagaya, T. Ikoma, T. Yoshioka and J. Tanaka, Chem. Commun., 2011, 47, 8430-8433.

8 M. Tagaya, S. Motozuka, T. Kobayashi, T. Ikoma and J. Tanaka, J. Mater. Chem., 2012, 22, 18741-18743.

9 M. Tagaya, N. Hanagata, T. Ikoma, T. Kobayashi, K. Shiba, T. Yoshioka and J. Tanaka, Key Eng. Mater., 2013, 529-530, 630-635.

10 M. Tagaya, T. Ikoma, Z. Xu and J. Tanaka, Inorg. Chem., 2014, 53, 6817-6827.

11 M. Tagaya, T. Ikoma, T. Yoshioka and J. Tanaka, J. Colloid Interface Sci., 2011, 363, 456-464.

12 A. S. Araujo and M. Jaroniec, J. Colloid Interface Sci., 1999, 218, 462-467.

13 A. S. Araujo and M. Jaroniec, Thermochim. Acta, 2000, 345, 173-177. 
14 V. C. Costa, M. J. Lochhead and K. L. Bray, Chem. Mater., 1996, 8, 783-790.

15 Q. Xu, L. Li, B. Li, J. Yu and R. Xu, Microporous Mesoporous Mater., 2000, 38, 351-358.

16 M. Tagaya, T. Ikoma, T. Yoshioka, F. Minami and J. Tanaka, Mater. Lett., 2011, 65, 2287-2290.

17 T. Kataoka, L. Y. Wang, K. Kobayashi, M. Nishikawa and M. Tagaya, Jpn. J. Appl. Phys., 2016, 55, 105503.

18 L. D. Deloach, S. A. Payne, W. L. Kway, J. B. Tassano, S. N. Dixit and W. F. Krupke, J. Lumin., 1994, 62, 85-94.

19 J. P. M. van Vliet and G. Blasse, Mater. Res. Bull., 1990, 25, 391-394.

20 M. Tachihante, D. Zambon, A. Arbus, M. Zahir, A. Sadel and J. C. Cousseins, Mater. Res. Bull., 1993, 28, 605-613.

21 R. Ternane, M. Trabelsi-Ayedia, N. Kbir-Ariguibb and B. Piriouc, J. Lumin., 1999, 81, 165-170.

22 W. D. Horrocks Jr and D. R. Sudnick, Acc. Chem. Res., 1981, 14, 384-392.

23 S. P. Mondejar, A. Kovtun and M. Epple, J. Mater. Chem., 2007, 17, 4153-4159.

24 A. Doat, M. Fanjul, F. Pellé, E. Hollande and A. Lebugle, Biomaterials, 2003, 24, 3365-3371.

25 T. Kataoka, K. Shiba and M. Tagaya, Colloid Interface Sci. Commun., 2016, 13, 1-5.
26 L. Li, Y. Liu, J. Tao, M. Zhang, H. Pan, X. Xu and R. Tang, J. Phys. Chem. C, 2008, 112, 12219-12224.

27 Y. Han, X. Wang, S. Li and X. Ma, J. Sol-Gel Sci. Technol., 2009, 49, 125-129.

28 S. Brunauer, P. H. Emmett and E. Teller, J. Am. Chem. Soc., 1938, 60, 309-319.

29 B. O. Fowler, Inorg. Chem., 1974, 13, 194-207.

30 K. C. Blakeslee and R. A. Condrate, J. Am. Ceram. Soc., 1971, 54, 559-563.

31 Y. Li and B. Yan, J. Solid State Chem., 2008, 181, 1032-1039.

32 V. Parvulescu and B. L. Su, Catal. Today, 2001, 69, 315-322.

33 E. P. Barrett, L. G. Joyner and P. H. Halenda, J. Am. Chem. Soc., 1951, 73, 373-380.

34 B. Szpikowska-Sroka, N. Pawlik, A. S. Swinarew and W. A. Pisarski, Ceram. Int., 2015, 41, 1167-11679.

35 H. Yang and Y. Kim, J. Lumin., 2008, 128, 1570-1576.

36 H. Weng and B. A. Yan, Sens. Actuators, B, 2016, 228, 702708.

37 H. Ikeda, T. Murata and S. Fujino, Opt. Mater., 2014, 36, 1119-1122.

38 Y. Nakamura, Am. Mineral., 1973, 58, 986-990.

39 E. Dowty, Am. Mineral., 1976, 61, 460-469. 